\title{
Recuperación post-fuego de la comunidad de líquenes epífitos en la provincia de Córdoba, Argentina
}

\author{
Juan Manuel Rodriguez ${ }^{1,2,3}$, Cecilia Estrabou ${ }^{1}$, Romina Fenoglio $^{1}$, Federico Robbiati $^{1}$, \\ María Cecilia Salas ${ }^{1}$ y Gonzalo Quiroga ${ }^{1}$
}

Recibida 14/08/2008. Aceptado 19/12/2008

\begin{abstract}
RESUMEN - (Recuperación post-fuego de la comunidad de líquenes epífitos en la provincia de Córdoba, Argentina). Los líquenes son reconocidos bioindicadores de la calidad del ambiente. Estudios de comunidades de líquenes en relación a los incendios forestales, han demostrado como responden al fuego por encima de las variables microambientales que modelan la comunidad en ausencia de este disturbio. El objetivo de la presente contribución es reconocer el efecto del fuego en la comunidad de líquenes del Bosque Serrano en Córdoba. Se seleccionaron dos zonas, una testigo y otra sometida a fuego en 1996. A través de un muestreo sistemático y estandarizado se relevó la presencia y cobertura de especies de líquenes en árboles y arbustos en las dos áreas estudiadas. Entre el área testigo y el incendiado la diversidad y cobertura de líquenes es similar pero varia su composición. Las especies en la zona incendiadas corresponden a líquenes heliófilos y adaptados a situaciones de estrés. El fuego como disturbio frecuente favorece la presencia de pocas especies con importantes coberturas que resistan las condiciones que el disturbio impone. Los incendios de alta intensidad y frecuencia dejan a la comunidad de líquenes sin posibilidades de desarrollo, disminuyendo la diversidad general y la calidad de los sistemas forestales en los que viven.
\end{abstract}

Palabras clave: bosque, comunidad liquénica, disturbio, fuego.

ABSTRACT - (Post-fire recovery of the epiphytic-lichen community in Cordoba province, Argentina). Lichens are recognized bioindicators of environmental quality. Studies of lichen communities in relation to forest fires have shown the response to fire over micro-environmental variables that shape the community in the absence of this disturbance. The aim of this contribution is to recognize the effect of fire on the lichen community of Sierra Chaco in Cordoba province. Two areas were selected, one without past fire and the other with fire in 1996. Coverage and presence of lichen species on trees and shrubs were recorded in the two areas using systematic, standardized sampling. Diversity and coverage of lichens is similar between the two areas but composition varies. The lichen species in the burned area are heliophilous and adapted to stress conditions. Fire as a frequent disturbance promotes the growth of few species with high coverage that are resistant to the conditions imposed by disturbance. High intensity and high frequency fires limit development opportunities for the lichen community thus diminishing overall diversity and quality of forest systems in which they live.

Key words: disturbance, fire, forest, lichen community

\section{Introducción}

En numerosos ecosistemas naturales el fuego es un factor recurrente que tiene alto impacto en el modelado del paisaje. $\mathrm{Su}$ efecto en los ecosistemas es considerable ya que modifica profunda y rápidamente los componentes estructurales del mismo. Este disturbio genera modificaciones en el seno de las comunidades vegetales que están fundamentalmente relacionadas a la resistencia de las distintas especies que la componen, a los mecanismos que pudieran haber desarrollado para sobrevivir a su paso y la posterior capacidad de las especies para recuperarse, y colonizar áreas post-fuego. Es por ello que los efectos del fuego sobre la vegetación pueden ser interpretados en términos de sucesión ecológica.

En particular, en nuestra provincia, el fuego afecta principalmente a bosques y pastizales, muchas veces con efectos irreversibles o de recuperación muy lenta. El estudio de las consecuencias del fuego y la recuperación de los ambientes luego de este disturbio permite contribuir a la elaboración de planes de manejo adecuados, minimiza las pérdidas ambientales y ayuda a la prevención de siniestros.

El Bosque Serrano comprende el primer piso de vegetación del sistema de sierras pampeanas, ubicado entre los 500 y los $1.300 \mathrm{~m}$ de altura aproximadamente. En Córdoba, se extiende entre los paralelos $31^{\circ}$ y $33^{\circ}$ y se halla caracterizado por un bosque generalmente abierto (Luti et al. 1979). Este ecosistema es uno de los más degradados y disturbados principalmente por el avance de la urbanización y los incendios forestales recurrentes (Cabido \& Zak 1999).

Los líquenes cumplen un papel importante en el funcionamiento del ecosistema siendo productores primarios, facilitadores de la formación de suelo, indicadores de la calidad del aire que los rodea así como también de la salud del bosque que los sustenta, proporcionan hábitat para pequeños animales y recursos para nidos y madrigueras, entre otros (Will-Wolf et al. 2002).

Los líquenes son organismos reconocidos como buenos bioindicadores de la calidad del ambiente. Por tal motivo, se los usa en ciudades como monitores de la calidad del aire (Hawksworth \& Rose 1970; Nimis et al. 2002), en sistemas forestales para evaluar del estado de los bosques (Tibell 1992; Zambrano García et al. 2000) y de los efectos del fuego (Mistry 1998; Boudreault et al. 2002). Estudios de comunidades de líquenes en relación a los incendios forestales destacan la respuesta de estos organismos como indicadores adecuados de este disturbio. Mistry (1998) demostró como los líquenes responden al fuego por encima de las variables microambientales que modelan la comunidad en ausencia de este disturbio. La recuperación de la o las

\footnotetext{
1 Universidad Nacional de Córdoba, Centro de Ecología y Recursos Naturales Renovables, Facultad de Ciencias Exactas, Físicas y Naturales, Córdoba, Argentina

2 CONICET - Doctorado en Ciencias Biológicas, Universidad Nacional de Córdoba

3 Autor correspondiente: juanmacor@yahoo.com.ar
} 
especies de líquenes va a depender de su forma de crecimiento, estrategia de dispersión y hábitat disponibles (Johansson et al. 2006).

Se han registrado alrededor de 50 especies de líquenes que forman parte del Bosque Serrano en la provincia de Córdoba (Estrabou \& García 1995; Estrabou et al. 2006). Algunos trabajos han contribuido al estudio de la diversidad y estructura de las comunidades liquénicas y su potencial bioindicador de disturbios ambientales (Estrabou et al. 2004; Estrabou et al. 2005).

En Argentina se han realizado investigaciones para conocer los efectos de los incendios forestales (Herrera et al. 1978; Martínez Carretero 1995; Verzino et al. 2005), pero pocas de ellas consideran a los organismos menos conspicuos (Abril \& González 1999), a pesar de que incrementan notablemente la diversidad de los ambientes y cumplen roles fundamentales en los procesos ecosistémicos. En este sentido, no se registran estudios sobre el impacto del fuego en la comunidad de líquenes, ni de su posible rol bioindicador luego de este disturbio. El objetivo del presente estudio es reconocer, a través de los cambios producidos en la comunidad de líquenes, un posible indicador del estado de recuperación post-fuego del bosque.

\section{Materiales y métodos}

Área de estudio - Sierras Chicas del norte de la Provincia de Córdoba, Argentina. El clima es templado, con temperatura media anual de aproximadamente $14{ }^{\circ} \mathrm{C}$, con máximas y mínimas absolutas de $41^{\circ} \mathrm{C}$ y $-9{ }^{\circ} \mathrm{C}$. Las precipitaciones alcanzan los $900 \mathrm{~mm}$ anuales y ocurren principalmente entre octubre y abril.

El ecosistema característico de las Sierras Chicas es el Bosque Serrano que se caracteriza por la dominancia del Lithraea ternifolia (Gillies ex Hook.) F.A.Barkley formando bosques más o menos densos en los faldeos más húmedos y frescos. Secundariamente Fagara coco (Gillies) Engl. in Engl \& Prantl. se ubica aislado entre los demás componentes en valles y llanos de altura otorgando un aspecto de sabana. Exceptuando las zonas de mayor altura y australes, alternando entre las especies mencionadas, podemos encontrar Condalia montana Castellanos, Prosopis alba Griseb., Acacia caven (Molina) Molina, entre otras (Luti et al. 1979; Cabido \& Zak 1999).

Contando con la ayuda de los registros de incendios de Bomberos Voluntarios de la localidad de La Granja (Córdoba-Argentina) se identificaron zonas de Bosque Serrano sometidas a fuego. Se seleccionó una zona control sin registro de incendio desde hace 50 años (Zona A) y una zona quemada hace 12 años (incendio en agosto de 1996) (Zona B). . Zona A (sin registro de incendios): $31^{\circ} 01^{\prime} 57^{\prime \prime} \mathrm{S} ; 64^{\circ} 16^{\prime} 01^{\prime \prime} \mathrm{O} ; 700-$ $800 \mathrm{msm}$. Bosque en buenas condiciones, constituido en su mayoría con ejemplares de Lithraea ternifolia de gran porte, y Condalia montana y Prosopis alba, que forman un dosel continuo, brindando elevada humedad y escasa luz al sotobosque.

- Zona B (incendio en 1996): $31^{\circ} 01^{\prime} 58^{\prime} \mathrm{S}$; 64¹7'20' O; 700 $800 \mathrm{msm}$. Bosque secundario con escasos árboles de gran porte y con dominio del estrato arbustivo (dominado por Acacia caven), restos de árboles y arbustos quemados sobre el suelo, fustes quemados de árboles en pie. Presenta condiciones físicas heterogéneas, escasa humedad, dispar incidencia de luz y suelo que alterna sectores sin coberturta vegetal y pastizales.

Métodos - cada una de las zonas fue geoposicionada y se delimitó un área de $100 \times 100 \mathrm{~m}$ en el centro del sector quemado. Dicha área fue dividida en 25 cuadrados de $20 \times 20 \mathrm{~m}$. En cada cuadrado se tomó el árbol y el arbusto más cercano al centro.

En un total aproximado de 50 árboles ( 25 por cada zona) se calculó la cobertura total de líquenes y de cada una de las especies, utilizando una grilla de $30 \times 20 \mathrm{~cm}$. Los censos se realizaron en la cara sudoeste del tronco (que es con frecuencia la única que presenta líquenes) y a 1,5 m del suelo (Estrabou \& García 1995; Estrabou et al. 2005).

Se censaron un total 50 arbustos (25 por cada zona), en cada uno se obtuvo una sección de la rama que cumpliera con los siguientes requisitos: portar líquenes, estar por encima del metro de altura, estar en la cara sudoeste del arbusto y tener más de $5 \mathrm{~cm}$ de diámetro. La cobertura de líquenes de cada sección de rama fue calculada mediante una grilla de $20 \times 5 \mathrm{~cm}$.

La determinación de las especies de líquenes se realizó en campo y laboratorio. Para ello se utilizó lupa, reacciones químicas de color, microscopio óptico y claves dicotómicas de identificación de especies (Scutari 1995a; b; Estrabou et al. 2006). En laboratorio se analizaron caracteres morfológicos, químicos y reproductivos para determinar especies. Se utilizó un estereomicrocopio ZEISS Stemy 2000-C y un microscopio óptico Bausch y Lomb RD para el estudio de los caracteres morfológicos y anatómicos de los ejemplares. Finalmente se siguieron técnicas de rutina en quimotaxonomía para la identificación química del material (White \& James 1985).

Análisis de datos - se consideró a cada uno de los árboles y arbustos seleccionados una unidad muestral. Una vez obtenidos todos los datos, se construyó una matriz de unidades por especies.

En cada zona se determinó el número de especies de líquenes según sean foliosos, fruticulosos, crustosos o cianolíquenes y además, se separaron de acuerdo a la estrategia reproductiva (asexual, sexual) siempre discriminando árboles y arbustos. Cabe destacar que los líquenes crustosos solo fueron considerados como grupo sin separar especies o géneros.

Para definir si las diferencias de cobertura liquénica entre árboles y arbustos de las dos zonas estudiadas eran estadísticamente significativas, se realizó la prueba $\mathrm{T}$ de diferencias de medias utilizando el programa INFOSTAT (ver.1.1). Previamente se verificó la normalidad de los datos analizados (Shapiro-Wilks modificado, 0.05 nivel de significación).

Mediante el programa PC-ORD (ver. 5.10) se aplicó el Análisis de Correspondencia Detendenciado (ACD) para árboles y para arbustos por separado.

A fin de poder comparar la riqueza y abundancia de especies en cada sitio de estudio, se calcularon los índices de diversidad de ShannonWiener (H') para cada una de las áreas. Se utilizó la formula $\mathrm{H}^{\prime}=\Sigma P_{i} \cdot \log _{2} P_{i}$ donde $\mathrm{P}_{\mathrm{i}}$ es la proporción de la i-esima especie sobre la sumatoria de la cobertura de todas las especies del área.

\section{Resultados}

Cobertura liquénica, composición y diversidad - la cobertura liquénica tanto en árboles como en arbustos no presenta diferencias significativas (Tab. 1). No obstante, existe una variación en la cobertura total promedio de líquenes en arbustos de la zona B (Fig. 1).

En total se registran 43 especies pertenecientes a 7 familias y a 17 géneros de líquenes (Tab. 2 y 3 ). El número de especies es mayor en los arbustos y árboles de la zona B (Fig. 2). El índice de Shannon-Wiener para cada zona, revela que la mayor diversidad se encuentra en los arbustos de la zona B $(4,1)$ y la menor diversidad se presenta en los árboles de la zona B $(3,24)$. Asimismo los árboles y arbustos de la zona A poseen valores intermedios (3,46 y 3,51 respectivamente).

Tabla 1. Test $\mathrm{t}$ de Student para la cobertura total relativa de líquenes para árboles y arbustos $(\mathrm{p}<0.05)$.

\begin{tabular}{lccc}
\hline & Valor $p$ & $g l$ & $t$ observado \\
\hline Árboles & 0,625 & 49 & $-0,64$ \\
Arbustos & 0,359 & 48 & $-0,93$ \\
\hline
\end{tabular}


Agrupamiento de las especies - el Análisis de Componentes Detendenciado realizado sobre la matriz de árboles por especies no arroja tendencias claras en el agrupamiento de las especies (Fig.3). El eje 1 muestra una tendencia a separar especies según el tipo de bosque en el

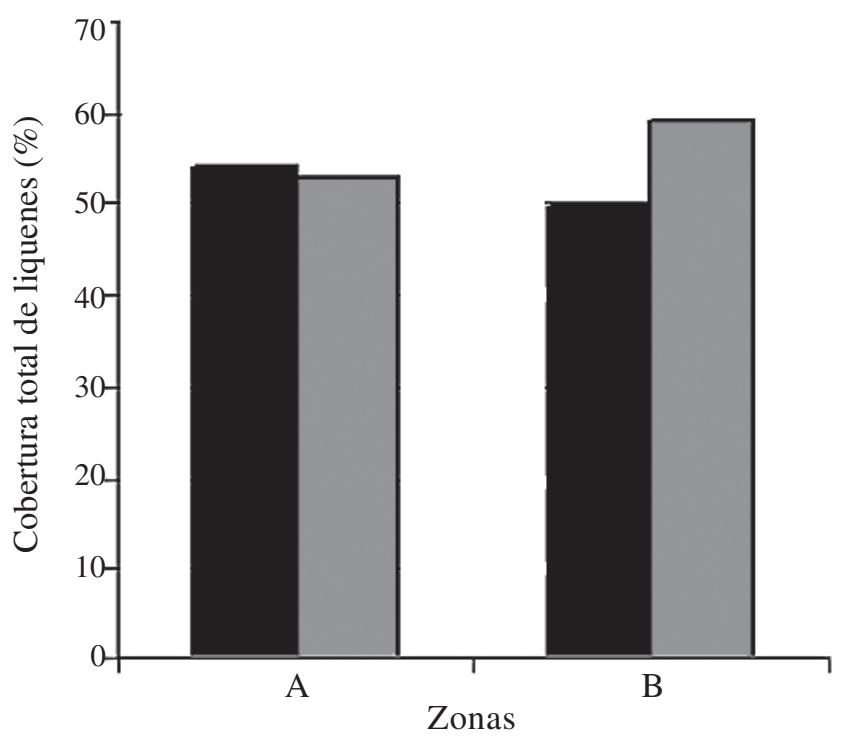

Figura 1. Cobertura total relativa promedio de líquenes para árboles y arbustos en las dos zonas estudiadas ( $\mathbf{\square}=$ árboles; $\mathbf{\square}=$ arbustos).

Tabla 2. Coberturas relativas promedio de las especies registradas en árboles para las dos zonas estudiadas. Las coberturas se expresan en porcentaje. Los líquenes crustosos son considerados como grupo, sin diferenciar especies.

\begin{tabular}{|c|c|c|}
\hline Especies & Zona A & Zona B \\
\hline Candelaria concolor (Dicks.). Stein & & 0,08 \\
\hline Canoparmelia crozalsiana (de Lesd.) Elix \& Hale & 12,08 & 7,40 \\
\hline Crustosos & 1,12 & 2,44 \\
\hline Dirinaria picta (Sw.) Clem. \& Shear & & 0,20 \\
\hline Heterodermia comosa (Eschw.) Follman \& Rédon & 0,04 & 0,04 \\
\hline H. obscurata (Nyl.) Trevis. & 6,96 & 8,84 \\
\hline Hyperphyscia granulata (Poelt) Moberg & 1,76 & 0,12 \\
\hline H. pandani (H.Magn.) Moberg & 3,60 & \\
\hline Hyperphyscia sp. & 0,56 & \\
\hline Leptogium sp. & 3,68 & 2,76 \\
\hline Myelochroa lindmanii (Lynge) Elix \& Hale & & 0,40 \\
\hline Normandina pulchella (Borrer) Nyl. & 1,00 & 1,92 \\
\hline Parmotrema austrosinense (Zahlbr.) Hale & 1,80 & 0,32 \\
\hline P. cetratum (Ach) Hale & 0,48 & \\
\hline$P$. conferendum Hale & 0,16 & \\
\hline $\begin{array}{l}\text { P. muelleri (Vain.) O. Blanco, A. Crespo, Divakar, } \\
\text { Elix \& Lumbsch }\end{array}$ & & 1,80 \\
\hline P. pilosum (Stizenb.) Krog \& Swinscow & 6,00 & 0,48 \\
\hline P. praesorediosum (Nyl.) Hale & & 1,80 \\
\hline$P$. reticulatum (Taylor) Choisy & & 13,20 \\
\hline Physcia aipolia (Humb.) Furner. & & 1,12 \\
\hline P. alba (Fée) Mull.Arg & & 3,60 \\
\hline P. erumpens Moberg & 0,24 & \\
\hline Physcia sp. & 0,24 & 0,04 \\
\hline P. tribacia (Ach.) Nyl. & 0,16 & \\
\hline P. undulata Moberg & 0,08 & \\
\hline Punctelia hypoleucites (Nyl) Krog. & & 1,00 \\
\hline P. microsticta (Müll. Arg.) Krog & 3,24 & \\
\hline$P$. perreticulata (Räsänen) & 1,88 & \\
\hline P. puntilla (Hale) Krog & 8,12 & 0,08 \\
\hline P. semansiana (W.L. Club \& C.F. Club.) Krog & 0,52 & \\
\hline Punctelia sp. & & 0,28 \\
\hline Usnea parvula Motyka & & 0,20 \\
\hline
\end{tabular}

Tabla 3. Coberturas relativas promedio de las especies registradas en arbustos para las dos zonas estudiadas. Las coberturas se expresan en porcentaje. Los líquenes crustosos son considerados como grupo, sin diferenciar especies.

\begin{tabular}{|c|c|c|}
\hline Especies & Zona A & Zona B \\
\hline Candelaria concolor (Dicks.). Stein & 0,61 & 0,74 \\
\hline Canoparmelia crozalsiana (de Lesd.) Elix \& Hale & 0,09 & 4,26 \\
\hline C. texana (Tuck.) Elix \& Hale & 1,00 & 0,70 \\
\hline Collema sp. & & 0,59 \\
\hline Crustosos & 10,30 & 4,33 \\
\hline Heterodermia albicans (Pers.) Swinscow \& Krog & & 0,07 \\
\hline H. comosa (Eschw.) Follman \& Rédon & 1,30 & 0,52 \\
\hline H. diademata (Taylor) D.D. Awasthi & 0,22 & \\
\hline H. obscurata (Nyl.) Trevis. & 2,09 & 1,67 \\
\hline Hyperphyscia granulata (Poelt) Moberg & & 0,04 \\
\hline H. pandani (H.Magn.) Moberg & 0,04 & \\
\hline Leptogium sp. & 2,65 & 0,37 \\
\hline Myelochroa lindmanii (Lynge) Elix \& Hale & 0,26 & 0,85 \\
\hline Parmotrema austrosinense (Zahlbr.) Hale & 1,61 & 4,11 \\
\hline P. cetratum (Ach) Hale & & 0,37 \\
\hline$P$. conferendum Hale & 6,44 & 4,15 \\
\hline P. consors (Nyl.) Elix \& Hale & & 0,82 \\
\hline P. chinanse (Osbeck) Hale \& Ahti & & 1,22 \\
\hline P. hababianun (Gylen.) Hale & 0,61 & 0,70 \\
\hline $\begin{array}{l}\text { P. muelleri (Vain.) O. Blanco, A. Crespo, Divakar, } \\
\text { Elix \& Lumbsch }\end{array}$ & & 1,22 \\
\hline P. pilosum (Stizenb.) Krog \& Swinscow & 13,78 & 11,30 \\
\hline P. praesorediosum (Nyl.) Hale & 0,48 & \\
\hline$P$. reticulatum (Taylor) Choisy & & 1,56 \\
\hline Phaeophyscia chloanta (Ach.) Moberg & 2,26 & 0,63 \\
\hline Physcia alba (Fée) Mull. Arg & & 0,19 \\
\hline P. tribacia (Ach.) Nyl. & & 2,96 \\
\hline Punctelia hypoleucites (Nyl) Krog. & 1,09 & 1,85 \\
\hline P. microsticta (Müll. Arg) Krog & 2,44 & 9,37 \\
\hline$P$. perreticulata (Räsänen) & & 0,59 \\
\hline P. puntilla (Hale) Krog & 0,30 & 0,19 \\
\hline P. semansiana (W.L. Club \& C.F. Club.) Krog & 2,78 & 0,93 \\
\hline P.a stictica (Delise ex Duby) Krog & & 0,44 \\
\hline Ramalina celastri (Spreng) Krog \& Swinscow & 0,87 & 0,48 \\
\hline Telochistes chrysophtalmmus (L.) Th.Fr. & 0,78 & 1,00 \\
\hline Usnea parvula Motyka & 0,57 & 1,07 \\
\hline Xanthoria parietina (L.) Th.Fr. & 0,26 & 0,04 \\
\hline
\end{tabular}

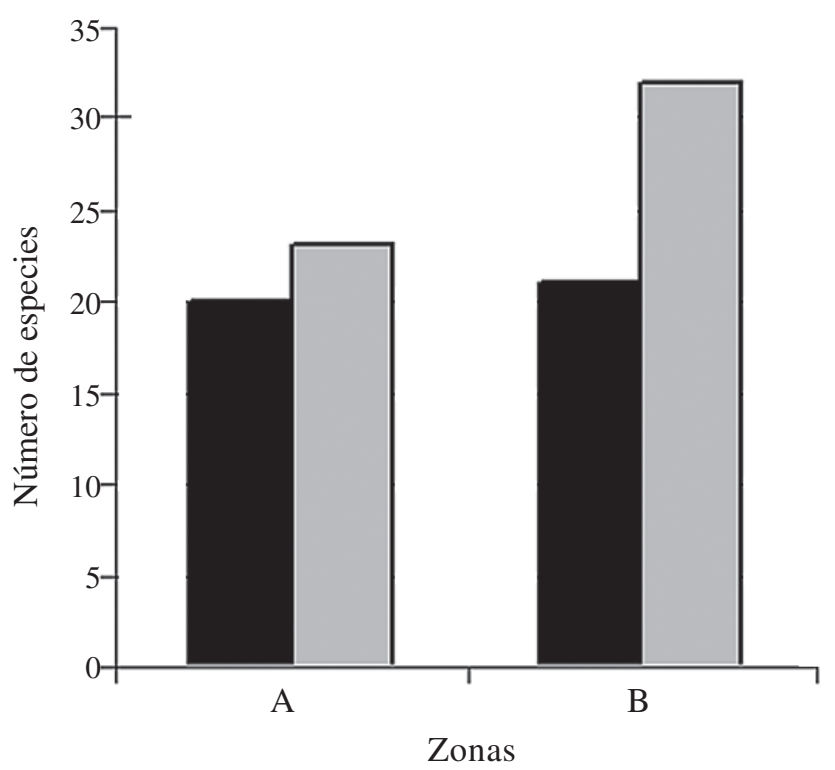

Figura 2. Número de especies total de líquenes para árboles y arbustos en las dos zonas estudiadas ( $=$ árboles; $=$ arbustos $)$. 


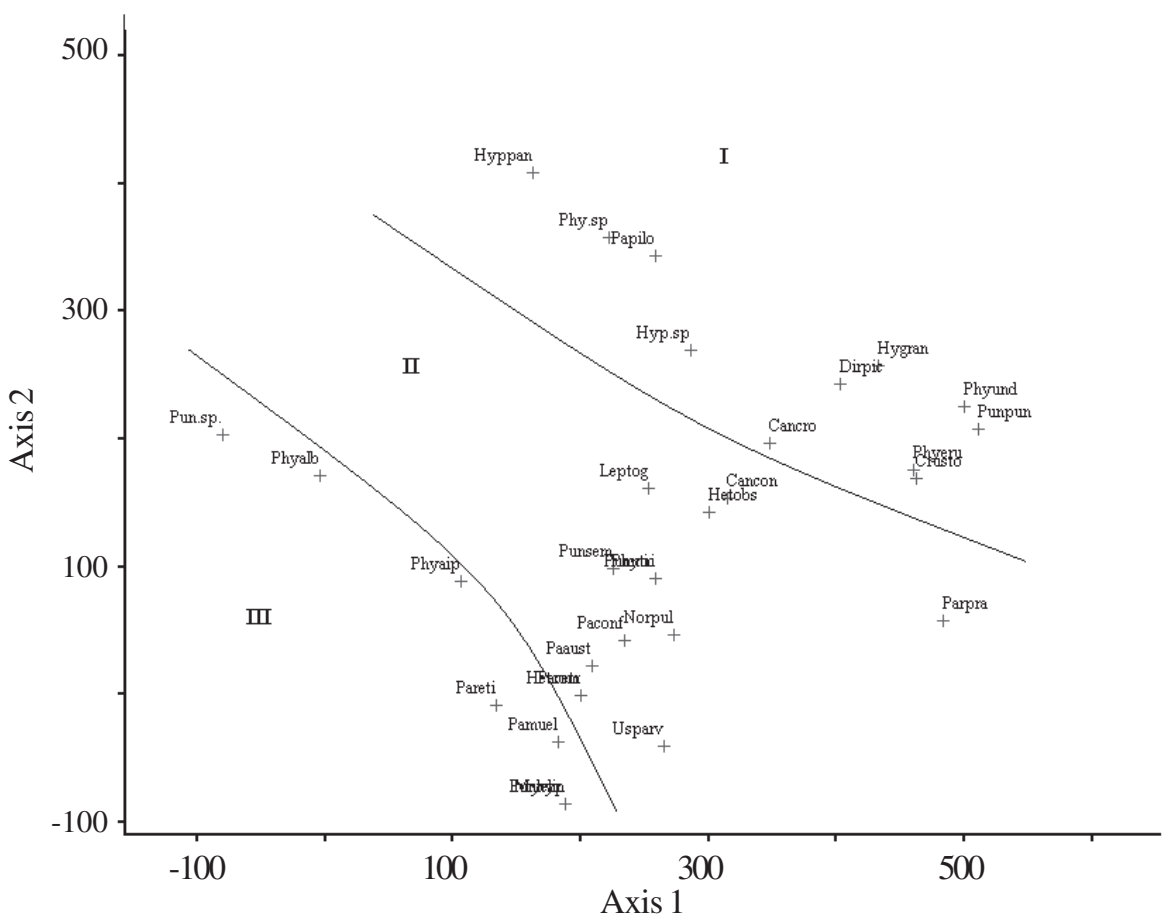

Figura 3. Análisis de Componentes Detendenciado para la matriz unidades de árboles vs cobertura relativa de especies. Abreviaturas: Cancon, Candelaria concolor; Cancroz, Canoparmelia crozalsiana; Crusto, Crustosos; Dirpic, Dirinaria picta; Hetcom, Heterodermia comosa; Hetobs; Heterodermia obscurata; Hypgra, Hyperphyscia granulata; Hyppan, Hyperphyscia pandani; Hyp.sp., Hyperphyscia sp.; Leptog., Leptogium sp.; Myelin, Myelochroa lindmani; Norpul, Normandina pulchella; Paaust, Parmotrema austrosinense; Pacetr, Parmotrema cetratum; Pamuel, Parmotrema muelleri; Papilo, Parmotrema pilosum; Paprae, Parmotrema praesorediosum; Pareti, Parmotrema reticulatum; Phyaip, Physcia aipolia; Phyalb, Physcia alba; Phyeru, Physcia erumpens; Phy.sp, Physcia sp.; Phytri, Physcia tribacia; Phyund, Physcia undulata; Punhyp, Punctelia hypoleucites; Punmic, Punctelia microsticta; Punper, Punctelia perreticulata; Punpun, Punctelia puntilla; Punsem, Punctelia semansiana; Pun.sp, Punctelia sp.; Usparv, Usnea parvula.

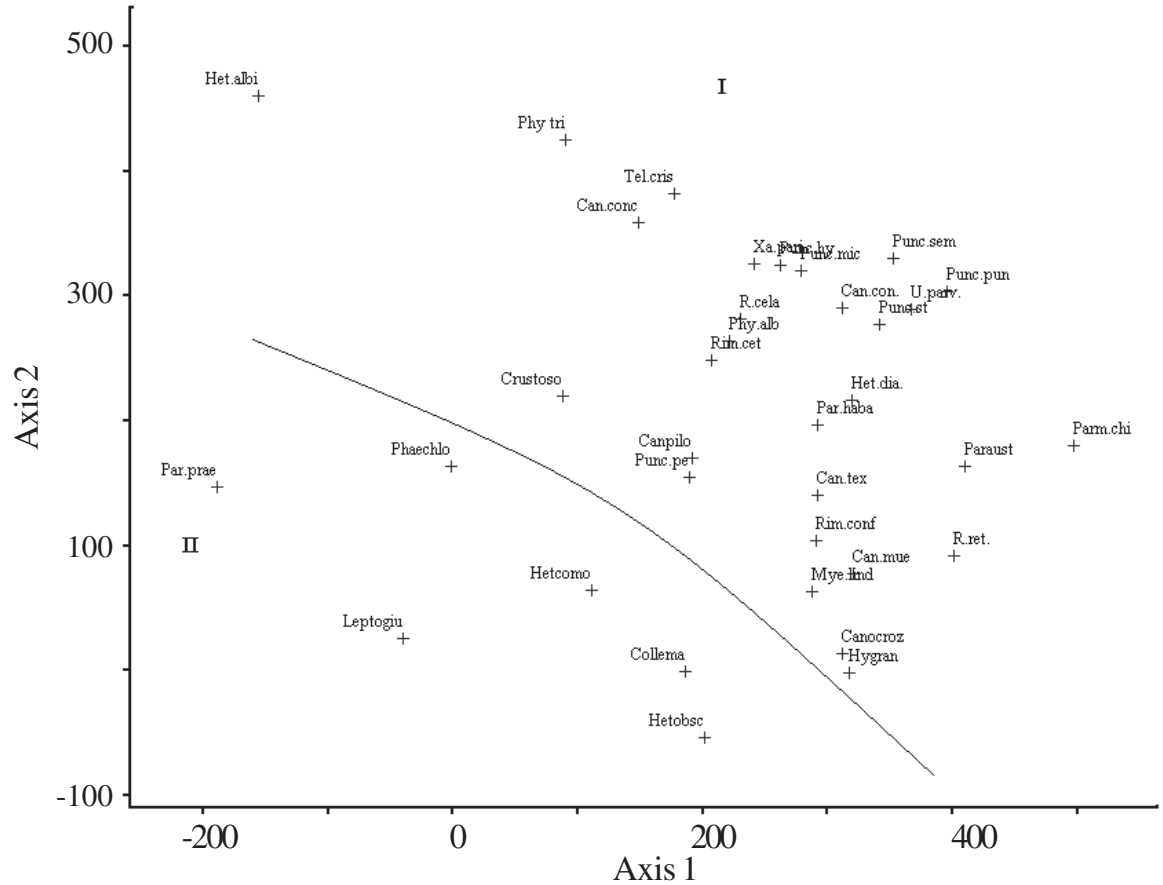

Figura 4. Análisis de Componentes Detendenciado para la matriz unidades de arbustos vs cobertura relativa de especies. Abreviaturas: Cancon, Candelaria concolor; Cancroz, Canoparmelia crozalsiana; Cantex, Canoparmelia texana; Collem, Collema sp.; Crusto, Crustosos; Hetalb, Heterodermia albicans; Hetcom, Heterodermia comosa; Hetdia, Heterodermia diademata; Hetobs; Heterodermia obscurata; Hypgra, Hyperphyscia granulata; Hyppan, Hyperphyscia pandani; Leptog., Leptogium sp.; Myelin, Myelochroa lindmani; Paaust, Parmotrema austrosinense; Pacetr, Parmotrema cetratum; Paconf, Parmotrema conferendum; Pacons, Parmotrema consors; Pamuel, Parmotrema muelleri; Papilo, Parmotrema pilosum; Paprae, Parmotrema praesorediosum; Pareti, Parmotrema reticulatum; Phachl, Phaeophyscia chloanta Phyaip, Physcia aipolia; Phyalb, Physcia alba; Phytri, Physcia tribacia; Punhyp, Punctelia hypoleucites; Punmic, Punctelia microsticta; Punper, Punctelia perreticulata; Punpun, Punctelia puntilla; Punsem, Punctelia semansiana; Punsti, Punctelia stictica; Ramcel, Ramalina celastri; Telcry, Teloschistes crysophtalmmus; Usparv, Usnea parvula; Xanpar Xanthoria parietina 
que se desarrollan. A la izquierda aparecen especies asociadas al bosque con disturbio, con especies de rápida dispersión en una comunidad menos estable en el tiempo. Hacia la derecha se concentran especies de una comunidad más estable en un bosque con mejores condiciones generales. Según la cobertura relativa y la posición de cada una de las especies en el gráfico de ACD se pueden diferencias tres agrupaciones. La primera (Fig. 3I) está representada por especies asociadas a los árboles de la zona A con coberturas importantes. Aquí podemos mencionar a Parmotrema pilosum, Canoparmelia crozalciana, Hyperphyscia spp. entre otras. El grupo de la Fig. 3II asocia especies con bajas coberturas o que se encuentran en ambas zonas como por ejemplo Heterodermia obscurata, Leptogium sp. El tercer grupo (Fig. 3III) presenta especies de la zona B como Parmotrema reticulatum, P. muelleri y Physcia alba.

Por otra parte el ACD realizado sobre la matriz de arbustos por especies muestra dos agrupaciones separadas sobre el eje 1, que discrimina de izquierda a derecha especies umbrófilas a heliófilas (Fig. 4). La primera agrupación estaría formada por especies asociadas exclusivamente en los arbustos de la zona A (Fig. 4I). Aquí se encuentran con elevada cobertura los líquenes crustosos y otros acompañantes como Leptogium sp., Heterodermia comosa y Phaeophyscia cloantha. En el segundo grupo (Fig. 4II) se agrupan las especies sin asociación clara con ninguna de las zonas y aquellas con coberturas mayores en la zona B: Canoparmelia crozalsiana, Parmotrema austrosinense, P. pilosum, P. reticulatum, Physcia spp. entre otras.

Bioforma y estrategias reproductivas - los líquenes foliosos dominan en las dos zonas estudiadas con respecto a las otras bioformas. Tres especies de líquenes fruticosos se registran para las comunidades de arbustos mientras que dos géneros de cianolíquenes se identifican en arbustos y sólo uno en árboles. En general predominan especies con tipos de reproducción asexual y mixta (Tab. 4).

Tabla 4. Número de especies según su estrategia reproductiva en árboles y arbustos para las 2 zonas estudiadas.

\begin{tabular}{cccc}
\hline & Estrategia Reproductiva & Árboles & Arbustos \\
\hline \multirow{2}{*}{ Zona A } & Sexual & 1 & 4 \\
& Asexual & 13 & 9 \\
& Mixtas & 6 & 10 \\
Zona B & Sexual & 3 & 6 \\
& Asexual & 6 & 14 \\
& Mixtas & 12 & 12 \\
\hline
\end{tabular}

\section{Discusión y conclusión}

Una de las variables que más influye en el desarrollo de una determinada comunidad de líquenes es la estructura y continuidad del bosque (Will-Wolf et al. 2002). El fuego como disturbio en los sistemas forestales estudiados cambia la estructura del bosque generando bordes, dejando parches, modificando la diversidad, eliminando por completo el estrato arbóreo y el arbustivo (Verzino et al. 2005). Estos cambios condicionan y regulan otras variables microclimáticas como son la luz, la humedad, la composición del aire que influyen directamente en la estructura de la comunidad de líquenes. Las comunidades de líquenes sobre árboles del bosque de las zonas A y B presentan una cobertura y diversidad similares, sin embargo, las diferencias entre estas zonas se dan principalmente en la composición de especies. En la zona B Parmotrema reticulatum es la especie dominante de una comunidad menos estable en el tiempo, Punctelia punctilla es especie acompañante, mientras que Canoparmelia crozalsiana queda relegada al tercer lugar en cobertura. Por otra parte en la zona A, una comunidad más estable, presenta a C. crozalsiana como la especie dominante. En un estudio realizado también en el Bosque Serrano, se determinó que $P$. reticulatum era la especie dominante en un ambiente de disturbio (Quiroga et al. 2008).

Cuando observamos en detalle los líquenes de la zona incendiada hace 12 años, podemos notar la necrosis en sectores del talo o quema parcial del mismo. Si el daño al liquen no es total, la porción del talo sobreviviente puede funcionar como colonizador de la nueva comunidad liquénica (Wolseley \& Aguirre-Hudson 1997). Entonces, la estructura de la comunidad en el sistema disturbado va a depender de las variables asociadas directamente al disturbio (intensidad del fuego, dirección, frecuencia, localización, etc.) que van a determinar algunas condiciones relacionadas con el sustrato, cambios en el microclima y los líquenes sobrevivientes, que aportarán propágulos para la recuperación de la comunidad.

Las comunidades de líquenes de árboles y arbustos son claramente diferentes. Si bien están reguladas por las mismas variables microclimáticas, las condiciones en uno y otro ambiente cambian y por lo tanto el impacto del disturbio también va a ser diferencial. La incidencia de luz y humedad sobre las ramas provoca condiciones óptimas para el desarrollo de los líquenes (Neitlich \& McCune 1997). En nuestro estudio de la comunidad liquénica de arbustos, a diferencia de los árboles, la mayor diversidad y cobertura se obtuvo en la zona B. Esta zona quemada hace 12 años presenta arbustos bajos a medianos (hasta 2 metros) que pueden crecer gracias a los espacios dejados por los árboles. Aquí las especies foliosas y heliófilas como Parmotrema pilosum y Punctelia spp. son dominantes en la comunidad.

Los arbustos de la zona A presentan varias especies de Punctelia cuya cobertura es muy baja en relación a la zona B mientras que Parmotrema pilosum también domina la comunidad. En los arbustos de esta zona es importante destacar la ausencia de Physcia spp., siendo especies de ambientes xéricos (Wolseley \& Aguirre-Hudson 1997) y la abundancia de Heterodermia spp. que presentan una marcada preferencia por zonas húmedas (Lücking et al. 2008).

La bioforma más frecuente son los líquenes foliosos tanto árboles como arbustos, estos resultados se corresponden con la tendencia general en el Bosque Serrano (Estrabou \& García, 1995). Los líquenes crustosos presentan la mayor cobertura en los arbustos de la zona testigo, patrón que coincide con 
Wolseley \& Aguirre Hudson (1997) quienes indican que este grupo tolera ambientes sombríos y húmedos.

Se conoce que las especies de reproducción asexual son más frecuentes en ambientes con disturbio (Mistry 1998) ya que los propágulos vegetativos se dispersan en grandes cantidades y colonizan los sustratos con mayor facilidad (Bowler \& Rundel 1975). En el presente estudio, hay una mayor proporción especies de reproducción mixta (apotecios y reproducción vegetativa) y asexual tanto en árboles como en arbustos para las zonas estudiadas.

La estructura y diversidad de la comunidad de líquenes asociada a los incendios forestales cambia en relación a la intensidad y frecuencia de este disturbio (Johansson et al. 2006). Un incendio con intensidad heterogénea puede abrir paso al desarrollo de nuevos y variados hábitat incrementando la diversidad local de líquenes. El fuego como disturbio frecuente va a favorecer el desarrollo de pocas especies con importantes coberturas que resistan las condiciones que el disturbio impone. Por último, los incendios forestales de alta intensidad y frecuencia dejan a la comunidad de líquenes y sus especies sin posibilidades de desarrollo disminuyendo la diversidad general y la calidad de los sistemas forestales en los que viven.

Es finalmente posible asociar una comunidad liquénica madura, caracterizada por las especies Canoparmelia crozalsiana, Leptogium sp. y especies crustosas, como indicadora de un tipo de bosque poco perturbado. Al mismo tiempo, la comunidad conformada por Parmotrema reticulatum y especies acompañantes, estaría relacionada con un bosque recientemente afectado por el fuego y modificado en su estructura.

\section{Agradecimientos}

Al Plan de Manejo del Fuego del gobierno de la provincia de Córdoba, Argentina por los fondos otorgados. Al Cuerpo de Bomberos Voluntarios de la localidad de La Granja por la información brindada.

\section{Referencias bibliográficas}

Abril, A. \& González, C. 1999. Dinámica de la fertilidad y de las poblaciones microbianas en suelos afectados por incendios en las Sierras de Córdoba (Argentina). Agriscientia 16: 63-70.

Boudreault, C.; Bergeron, Y.; Gauthier, S. \& Drapeau, P. 2002. Bryophyte and lichen communities in mature to old-growth stands in eastern boreal forests of Canada. Canadian Journal of Forest Research 32: 1080-1093.

Bowler, P.A. \& Rundel, P.W. 1975. Reproductive strategies in lichens. Botanical Journal of Linnean Society 70: 325-340.

Cabido, M.R. \& Zak, M.R. 1999. Vegetación del norte de Córdoba. Secretaría de agricultura, ganaderia y recursos renovables de la provincia de Córdoba y Agencia Córdoba ambiente. Córdoba.

Estrabou, C. \& García, L. 1995. Comunidades liquénicas cortícolas sobre Lithraea ternifolia en las Sierras Chicas de la provincia de Córdoba, Argentina. Botánica Complutensis 20: 35-43.

Estrabou, C.; Stiefkens, L.; Hadid, M.; Rodríguez, J.M. \& Pérez, A. 2004. Effects of air pollutants on morphology and reproduction in four lichen species. Ecología en Bolivia 39: 33-45.
Estrabou, C.; Stiefkens, L.; Hadid, M.; Rodríguez, J.M. \& Pérez, A. 2005. Estudio de la comunidad liquénica en cuatro ecosistemas de la provincia de Córdoba. Boletín de la Sociedad Argentina de Botánica 40: 3-12.

Estrabou, C.; Rodríguez, J.M.; Prieri, B.; \& Lijteroff, R. 2006. Contribución al conocimiento de los líquenes epifitos del extremo sur del Gran Chaco. Kurtziana 32: 25-43.

Hawksworth, D.L. \& Rose, F. 1970. Qualitative scale for estimating sulphur dioxide air pollution in England and Wales using epiphytic lichens. Nature 227: 145-148.

Herrera, M.; Bertrán, M.; Galera, F.M.; Luti, R. \& Menghi, M. 1978. Incendio y pastoreo en estepas de altura de las Sierras Chicas de Córdoba. Ecología 3: 95-99.

Johansson, P.; Wetmore, C.M.; Carlson, D.J.; Reich, P.B. \& Thor, G. 2006. Habitat preference, growth form, vegetative dispersal and population size of lichens along a wildfire severity gradient. The Bryologist 109: 527-540.

Lücking, R.; del Prado, R.; Lumbsch, T.H.; Will-Wolf, S.; Aptroot, A.; Sipman, H.J.M.; Umaña, L. \& Chaves, J.L. 2008. Phylogenetic patterns of morphological and chemical characters and reproductive mode in the Heterodermia obscurata group in Costa Rica (Ascomycota, Physciaceae). Systematics and Biodiversity 6: $31-41$

Luti, R.; Solis, A.; Galera, F.M.; Berzal, M.; Nores, M.; Herrera, M. \& Barrera, J. 1979. Vegetación. Pp. 297-367. In: J.B. Vázquez; R. Miatello \& M. Roqué (eds.). Geografía Física de la Provincia de Córdoba. Buenos Aires, Editorial Bolt.

Martínez Carretero, E. 1995. Los incendios forestales en la Argentina. Multequina 4: 105-114.

Mistry, J. 1998. Corticolous lichens as potential bioindicators of fire history: a study in the cerrado of the Distrito Federal, central Brazil. Journal of Biogeography 25: 409-441.

Nimis, P.L.; Scheidergger, C. \& Wolseley, P.A. 2002. Monitoring with Lichens - Monitoring Lichens. Netherlands, Kluwer Academic Publishers.

Neitlich, P.N. \& McCune, B. 1997. Hotspots of epiphytic lichen diversity in two young managed forests. Conservation Biology 11: $172-182$.

Quiroga, G.; Estrabou, C. \& Rodriguez, J.M. 2008. Lichen community response to different management situations in a protected forest of Córdoba, Argentina. Lazaroa 29: 131-138.

Scutari, N. 1995a. Los macrolíquenes de Buenos Aires, I: Phaeophyscia, Physcia y Pyxine (Physciaceae, Ascomycota). Darwiniana 33: 149-176.

Scutari, N. 1995b. Los macrolíquenes de Buenos Aires, I: Dirinaria, Heterodermia e Hyperphyscia (Physciaceae Ascomycotina). Darwiniana 33: 211-231.

Tibell, L. 1992. Crustose lichens as indicators of forest continuity in boreal coniferous forests. Nordic Journal of Botany 12: 427-450.

Verzino, G.; Joseau, J.; Dorado, M.; Gellert, E.; Rodríguez Reartes, S. \& Nóbile, R. 2005. Impacto de los incendios sobre la diversidad vegetal, sierras de Córdoba, Argentina. Ecología Aplicada 4: 25-34.

White, F.J. \& James, P.W. 1985. A new guide to microchemical tecniques for the identification of lichen substances. Bulletin of British Lichen Society 57: 1-41.

Will-Wolf, S.; Esseen, P.A. \& Neitlich, P. 2002. Monitoring Biodiversity And Ecosystem Function: Forests. Pp. 203-222. In: P.L. Nimis; C. Scheidegger \& P.A. Wolseley (eds.). Monitoring with Lichens - Monitoring Lichens. Netherlands, Kluwer Academic Publishers.

Wolseley, P.A. \& Aguirre-Hudson, B. 1997. Fire in tropical dry forests: corticolous lichens as indicators of recent ecological changes in Thailand. Journal of Biogeography 24: 345-362.

Zambrano García, A.; Nash, T.H. \& Herrera-Campos, M.A. 2000. Lichen decline in desierto de los leones (Mexico City). The Bryologist 103: 428-441. 